\title{
Plant-pathogen interactions: toward development of next-generation disease-resistant plants
}

\begin{abstract}
Briskly evolving phytopathogens are dire threats to our food supplies and threaten global food security. From the recent advances made toward high-throughput sequencing technologies, understanding of pathogenesis and effector biology, and plant innate immunity, translation of these means into new control tools is being introduced to develop durable disease resistance. Effectoromics as a powerful genetic tool for uncovering effector-target genes, both susceptibility genes and executor resistance genes in effector-assisted breeding, open up new avenues to improve resistance. TALENs (Transcription Activator-Like Effector Nucleases), engineered nucleases and CRISPR (Clustered Regulatory Interspaced Short Palindromic Repeats)/Cas9 systems are breakthrough and powerful techniques for genome editing, providing efficient mechanisms for targeted crop protection strategies in disease resistance programs. In this review, major advances in plant disease management to confer durable disease resistance and novel strategies for boosting plant innate immunity are highlighted.
\end{abstract}

Keyword: CRISPR/Cas9; Effector-assisted breeding; Effectoromics; Genome editing; Immune-priming compounds; Novel resistance strategies; TALENs 